\title{
Genomic associations with poxvirus across divergent island populations in Berthelot's pipit
}

\author{
Eleanor Sheppard ${ }^{1}$, Claudia Martin ${ }^{1}$, Claire Armstrong${ }^{1}$, Catalina González-Quevedo ${ }^{1}$, \\ Juan Carlos Illera ${ }^{2}$, Alexander Suh ${ }^{1}$, Lewis Spurgin ${ }^{1}$, and David Richardson ${ }^{1}$ \\ ${ }^{1}$ University of East Anglia \\ ${ }^{2}$ Universidad de Oviedo
}

December 17, 2021

\begin{abstract}
Understanding the mechanisms and genes that enable animal populations to adapt to pathogens is important from an evolutionary, health and conservation perspective. Berthelot's pipit (Anthus berthelotii) experiences extensive and consistent spatial heterogeneity in avian pox infection pressure across its range of island populations, thus providing an excellent system with which to examine how pathogen-mediated selection drives spatial variation in immunogenetic diversity. Here we test for evidence of genetic variation associated with avian pox at both an individual and population-level. At the individual level, we find no evidence that variation in MHC class I and TLR4 (both known to be important in recognising viral infection) was associated with pox infection within two separate populations. However, using genotype-environment association (Bayenv) in conjunction with genome-wide (ddRAD-seq) data, we detected strong associations between population-level avian pox prevalence and allele frequencies of single nucleotide polymorphisms (SNPs) at a number of sites across the genome. These sites were located within genes involved in cellular stress signalling and immune responses, many of which have previously been associated with responses to viral infection in humans and other animals. Consequently, our analyses provide evidence that pathogen-mediated selection has shaped genomic variation among relatively recently colonised island bird populations, and highlights the utility of genotype-environment associations for identifying candidate genes involved in adaption to local pathogen pressures.
\end{abstract}

\section{Hosted file}

Genomic associations with poxvirus across divergent island populations in Berthelot's pipit.docx available at https://authorea.com/users/451619/articles/549844-genomic-associations-withpoxvirus-across-divergent-island-populations-in-berthelot-s-pipit 